p16 gene is increasingly accompanying the severity of lesions, but without statistical relevance.

\section{P3.209 AGE DIFFERENCES BETWEEN HETEROSEXUAL PARTNERS: IMPLICATIONS FOR THE SPREAD OF CHLAMYDIA TRACHOMATIS}

${ }^{1} \mathrm{JH}$ Smid, ${ }^{1} \mathrm{~V}$ Garcia, ${ }^{2} \mathrm{CH}$ Mercer, ${ }^{1} \mathrm{~N}$ Low, ${ }^{1} \mathrm{CL}$ Althaus. ${ }^{1}$ Institute of Social and Preventive Medicine, University of Bern, Bern, Switzerland; ${ }^{2} U C L$ Research Department of Infection and Population Health, University College London, London, UK

\subsection{6/sextrans-2017-053264.444}

Introduction The consequences of mixing between sexual partners of different ages on the transmission and prevalence of sexually transmitted infections (STI) are still not well understood. Using sexual behaviour data, we obtained a detailed quantitative description of sexual mixing by age. We then investigated the impact of age mixing on the age- and sex-specific prevalence and incidence of Chlamydia trachomatis $(\mathrm{Ct})$ using a dynamic transmission model.

Methods First, we used age- and sex-specific data about the proportion of individuals who are sexually active, sexual partner change rates, and the ages of the three most recent partners at first episode of sexual intercourse from the second and third British National Surveys of Sexual Attitudes and Lifestyles (Natsal-2 and Natsal-3). We used a parametric description of the partner ages using skew-normal distributions and combined the data to reconstruct age mixing between heterosexual partners. Second, we incorporated the mixing patterns into a compartmental transmission model to investigate the age groups amongst which $C t$ is most likely to be transmitted and in which direction.

Results On average, males reported sex with younger female partners (median age difference -5.3 ; IQR [-1.6,-8.9] years) and females reported having male partners of similar age (median age difference -0.4 ; IQR $[-2.7,1.9]$ years). The median and the skewness of partner age distributions depend heavily on the age of the respondent. Ct-transmitting partnerships are typically between an older male and a younger female partner. In $60 \%$ of $C t$-transmitting partnerships, at least one partner was $>25$ years old.

Conclusion Our study illustrates the importance of sexual mixing patterns on $C t$ spread and indicates that a majority of transmitted infections are in age groups outside of those included in typical $C t$ screening programs. Our method for the incorporating sexual behaviour data into dynamic transmission models can be used to study the transmission of any STI and to understand the potential impact of control strategies that target specific age groups.

\section{P3.210 ESTIMATING THE ANTIBODY PREVALENCE OF HERPES SIMPLEX VIRUS TYPE 2 AMONG SELECT MIDDLE EAST AND NORTH AFRICA POPULATIONS}

${ }^{1}$ Soha Dargham, ${ }^{2}$ Gheyath Nasrallah, ${ }^{2}$ Enas Al-Absi, ${ }^{2}$ Layla Mohammed, ${ }^{2}$ Rana Al Disi, ${ }^{2}$ Mariam Noufal, 'Laith Abu-Raddad. 'Weill Cornell Medicine-Qatar, Doha, Qatar; ${ }^{1}$ Qatar University, Doha, Qatar

\subsection{6/sextrans-2017-053264.445}

Introduction There are very limited data on herpes simplex virus type 2 (HSV-2) infection in the Middle East and North Africa (MENA). We examined the overall and age-specific
HSV-2 antibody prevalence among select MENA populations currently residing in Qatar.

Methods Sera were collected from blood donors attending Hamad Medical Corporation June 2013-2015. Specimens were screened for HSV-2 antibodies using HerpeSelect 2 ELISA IgG kits. All positive and equivocal specimens detecting presence of HSV-2 antibodies were retested for final HSV-2 status using Euroline Westernblot assays. Demographic information included nationality, age, and sex. Age was grouped into 8 bands: $\leq 24,25-29,30-34,35-39,40-44,45-49,50-$ 54 , and $\geq 55$. Age-specific trend of HSV-2 infection among Egyptian, Fertile Crescent (Iraq, Jordan, Lebanon, Palestine, and Syria) and Qatari nationals was described and assessed using the Cochran-Armitage test.

Results Sera from 2165 blood donors were tested for HSV-2. Among 132 retested specimens, 66 were confirmed positive. Country-specific HSV-2 prevalence was measured at $5.5 \%$ (95\% confidence interval (CI) 3.7\%-8.2\%) for Qataris, $4.5 \%$ (95\%CI 1.9\%-10.0\%) for Iranians, 4.2\% (95\%CI 1.8\%-9.5\%) for Lebanese, 3.1\% (95\%CI 1.2\%-7.7\%) for Sudanese, 3.0\% (95\%CI 1.4\%-6.4\%) for Palestinians, 2.2\% (95\%CI $1.1 \%$ $4.3 \%$ ) for Egyptians, 2.0\% (95\%CI 1.0\%-5.0\%) for Syrians, $1.0 \% \quad(95 \% \mathrm{CI} \quad 0.3 \%-3.6 \%)$ for Jordanians, $0.7 \% \quad(95 \% \mathrm{CI}$ $0.1 \%-3.7 \%$ ) for Yemenis, and $0.5 \%$ (95\%CI $0.1 \%-2.8 \%$ ) for Pakistanis. Age-specific HSV-2 prevalence was estimated for Egyptians, nationals of the Fertile Crescent, and Qataris. Overall, HSV-2 prevalence increased with age, but the trend was not always statistically significant in these populations. HSV-2 prevalence was significantly higher for females at 9.1\% $(95 \%$ CI $4.7 \%-16.9 \%)$ than males at $2.8 \%$ (95\%CI $2.2 \%-3.6 \%$ ) $\left(\chi^{2}\right.$ p-value $\left.<0.01\right)$.

Conclusion HSV-2 prevalence among MENA nationals was found to be lower than that commonly found in other regions. However, these observed prevalence levels suggest unmet needs for sexual health and control of sexually transmitted infections (STIs) transmission. Programs need to be established to tackle STIs and their disease burden in this region.

\section{P3.211 PROFILE OF MEN WITH HIV+ INFECTION DIAGNOSIS: A COMPARATIVE STUDY ON A PUBLIC HEALTH SERVICE BETWEEN THE YEARS 2003 AND 2013}

${ }^{1} \mathrm{GF}$ Souza, ${ }^{1} \mathrm{~A}$ Almeida, ${ }^{2} \mathrm{HLB}$ Reis, ${ }^{3} \mathrm{HRA}$ Montenegro, ${ }^{4} \mathrm{MRL}$ Passos, ${ }^{5} \mathrm{DC}$ Ferreira. ${ }^{1}$ UNIABEU, Belford Roxo - RJ, Brazil; ' UFES, Vitoria - ES, Brazil; ${ }^{3}$ UFRJ, Rio de Janeiro - RJ, Brazil; ${ }^{4}$ Prof Titular E Chefe do Setor de DST UFF, Niteroi-RJ, Brazil; ${ }^{5}$ UNESA, UVA, Rio de Janeiro - RJ, Brazil

\subsection{6/sextrans-2017-053264.446}

Introduction To describe and compare the profile of men who had a positive diagnosis to the infection by the Human Immunodeficiency Virus (HIV) at a health service in the municipality of Nova Iguaçu in Baixada Fluminense, Rio de Janeiro, in the years 2003 and 2013.

Methods Comparative and retrospective study in which the patient's medical records were utilised in the referred diagnostic service.

Results In 2003, 772 men were attended, and $116(15.02 \%)$ were diagnosed with HIV, among them, 41 (35.3\%) had 1 to 3 years of complete school education, $26(22.4 \%)$ had 4 to 7 years of school education and $26(22.4 \%)$ had from 8 to 11 years. $20(17.2 \%)$ individuals had STDs in the past year. 33 (28.4\%) individuals made regular use of condoms and 52 men 